\title{
Next-Generation Sequencing Studies: Optimal Design and Analysis, Missing Heritability and Rare Variants
}

\author{
Paul Marjoram • Duncan C. Thomas
}

Published online: 23 September 2014

(C) Springer International Publishing AG 2014

\begin{abstract}
As we enter the "Post Genome-wide Association Study" era, there is a widespread desire to move towards sequencing study samples, often after an initial genomewide association analysis that has found some loci associated with a phenotype of interest in a given population. The rationale is clear: the initial study is highly unlikely to have found all, or even most, associated loci. Indeed, in terms of heritable variation, for virtually every phenotype, much remains to be explained. The details of how such a sequence-based study should be designed are unclear. In this article we review the rationale for such a sequence-based approach, such as the desire to find new (likely rare) variants that are also associated with the phenotype, and the wish to explain the "missing heritability". We go on to review simulation-based and databased ways of developing guidelines for the design of such next-generation sequence-based studies. Finally, we discuss the desirability of using biological prior information to help lessen the problems caused by "the curse of dimensionality" in this context.
\end{abstract}

Keywords GWAS · Next-generation sequencing · Study design $\cdot$ Rare variants $\cdot$ Missing heritability

\section{Introduction}

Good data are catalysts for good science. Good data are themselves generally a consequence of good (and frequently new) technology. Indeed, in his best seller "The Scientists", John Gribbin asserts that "It is the luck of the draw, or

P. Marjoram $(\bowtie) \cdot$ D. C. Thomas

Department of Preventive Medicine, Keck School of Medicine,

University of Southern California, $2001 \mathrm{~N}$. Soto Street, C-202V, MC

9234, Los Angeles, CA 90089-9234, USA

e-mail: pmarjora@usc.edu historical accident, who gets remembered as the discoverer of a new phenomenon. What is more important than human genius is the development of technology [...]". In the biological sciences we are lucky enough to live in a period in which a wide variety of technological revolutions are occurring. These are allowing us to collect data that are far richer than those that have been available in the past. For example, it is now possible to collect large quantities of sequence data for thousands of individuals. Data of this sort are a gold mine in our efforts to understand the relationship between our genetic and phenotypic makeups, and they will likely allow for the nomination of more "accidental geniuses."

A recent focus of these technological advances has been genome-wide association studies [GWAS]. We have collected data on hundreds of thousands of common genetic polymorphisms for large samples of individuals in relation to diseases of interest. The hope has been that these data may hold the key to understanding the genetic basis of common human diseases. This hope was reflected, and rewarded, by a number of early success stories, [e.g., 1-8], and ultimately we have found many hundreds of loci associated with a variety of diseases. So far, so good. However, for a number of reasons it has become clear that the picture is rather more nuanced than we anticipated. While finding large numbers of associated variants is clearly a significant advance in our knowledge, the usefulness of that advance is dependent upon how much of the phenotypic variation is explained by the detected associated variants.

The "poster child" for this issue is the search for loci associated with human height. Here, sample sizes are unusually large compared to traditional GWAS (since such analyses of height are generally meta-studies), which leads to unusually high power and uncovering unusually large numbers of associated loci. For example, Lango Allen et al. [9], reports that there are over 180 loci associated with height. However, the concern is that variation in human height is known to be about 
75-80 \% heritable [10-12], whereas Lango Allen et al., estimate the proportion of variation in height explained by all discovered loci, in toto, to be about $10 \%$. Thus, much of the heritable variation has yet to be explained. This phenomenon is known as missing heritability, and it has led to some soul searching regarding the future of GWAS.

A variety of possible explanations have been proposed for missing heritability [13•]. We summarize a number of these below:

\section{Rare Variants}

GWAS exploit a variety of platforms that allow the interrogation of large numbers of single nucleotide polymorphism [SNPs] for large numbers of individuals. Because of constraints such as cost, and technological restrictions on the hardware involved, just a subset of all known variants can be included on such platforms. Therefore, for good reason, it has been typical to populate these platforms with common SNPs (greater than $1 \%$ population frequency, say). This provides more "bang for the buck", since the inclusion of rare variants would lead to many SNPs that appeared to be nonpolymorphic in any given study sample.

However, if rare variants explain a substantial amount of phenotypic variation, this choice in a GWAS platform design means that we have been deliberately excluding the very SNPs that explain much phenotypic variation. There has been some discussion of the extent to which we can expect common SNPs to "tag" rare variants, and, thereby, hope to see associations in such tagging common SNPs even when the truly causative SNP is rare [e.g.,14, 15]. The overwhelming reaction to this realization, however, has been to want to interrogate rare variants directly. This is made possible due to another recent technological revolution: our ability to collect sequence-level data.

\section{Aggregations of Variants of Small Effect}

Various analyses of psychiatric diseases [16] and analyses of height including those discussed above [9, 17], have suggested that very many variants may be contributing to the variation in these traits. For example, Purcell et al.'s analysis of polygenic variation in schizophrenia and bipolar disorder [1] showed that adding more and more weakly associated SNPs to a polygenic model continued to improve risk prediction even up to a significance threshold of $p<0.50$ ! As noted above, Lango Allen et al., found 180 associated loci that collectively explained $10 \%$ of the phenotypic variation in height and, extrapolating from the available sample size of 183,000 individuals, predicted that increasing the sample size to about 500,000 would allow detection of additional loci that would increase the explained variance to $15 \%$. Using a random effects model, however, Yang et al. found that using all 300,000 common variants, and not just those that were individually significantly associated, could explain as much as $45 \%$ of the variance in height. This is part of a growing view that it may be aggregations of common variants of small effect, rather than rare variants, that explain most of missing heritability [e.g. 18•, 19••].

The work of $[16,17]$ illustrates the two main approaches that have been taken recently for the aggregation analysis of rare variants, for which conventional logistic models are inadequate because of the twin problems of extreme sparseness and multiple comparisons. The first of these is burden testing (e.g., [20]) based on a 1-degree of freedom test of association with some form of weighted sum of allele counts. The second is variance components tests (equivalently, random effects models) that test for overdispersion of risks across a group of variants rather than a systematic departure from the null in the same direction. The first of this class of methods is Neyman's [21] $\mathrm{C}_{\alpha}$ test of overdispersion, first proposed for rare variants by Neale [22]. The most popular of the random effects tests, however, is based on relating the similarity of phenotypes to the similarity of genotype vectors across pairs of apparently unrelated individuals, the so-called Sequence Kernel Association Test (SKAT) [23, 24], or across family members [25•, 26, 27]. Hierarchical modeling [28] provides yet a third approach, based on treating the relative risk coefficients from a conventional logistic regression on individual SNPs or rare variants as random variables in a second level model, where the focus is on their overall distribution or associations with external annotation information. We defer further discussion of this approach to later.

\section{Heritability Definitions}

When comparing the phenotypic variation explained by known genetic polymorphisms to the total phenotypic variation believed to be heritable, it is important to make sure we are not comparing "apples" to "oranges". Specifically, GWAS analyses typically look for marginal associations, and explore the extent to which additive effects can explain phenotypic variation. In other words, they try to explain "narrow sense" heritability. However, heritability is often quantified in the "broad sense", which also includes epistatic effects. In a recent paper, Zuk et al. [29••] explored this issue, arguing that much of the purported missing heritability might be due to so-called "phantom heritability", caused by a component of heritability attributable to epistatic interactions. They then proposed a method for estimating additive heritability that was not subject to inflation due to genetic interactions, and would, therefore, allow a fairer comparison between actual and explained heritability. 
Disease is Complex and GWAS is Difficult:

The use of Biological Priors

Much disease is complex, and will obviously have many underlying factors and causes. These may be captured in epistatic effects, for example, as discussed above. Traditional GWAS users are not ignorant of the likelihood of epistasis, but choose to focus on marginal effects. This is an implicit recognition of the lack of power that results in this context if one searches for interactions in addition to marginal effects. The multiple comparison penalty becomes a much more significant obstacle if we search all interactions. Furthermore, perhaps it is not reasonable to treat all possible interactions, or even marginal effects, equally. As George Orwell might say "All effects are possible, but some are more possible than others".

As one approach to dealing with the extremely high dimensionality of an exhaustive search for epistatic interactions (of the order of a trillion comparisons for all possible 2-way interactions just among common SNPs), Lewinger et al. [30] adapted earlier 2-step approaches for gene-environment interactions. They used a preliminary screening step, that is statistically independent of the case-control test for interaction, to identify potential interactions and reduce the multiple testing penalty. They showed that this approach can be considerably more powerful than exhaustive testing. Other approaches have aimed at using various network methods to explore higher order interactions [31-33]. For rare sequence variants, testing for individual variants, let alone their interactions, is likely to be under-powered, so the focus has shifted to various forms of regional associations (e.g., the burden or SKAT tests discussed above). Adding region-by-region interactions seems like a logical extension, but to our knowledge has not yet been explored.

One can also use biological information to inform analyses of sequence level data, in which the curse of dimensionality is likely to be substantially worse than in GWAS data in several ways. We discuss this more fully later.

\section{Design of Next-Generation Sequencing Follow-up to GWAS}

One strategy for follow-up to GWAS is complete genome sequencing of all members of a study population. As costs fall, this may become the default strategy but, for now, more subtlety of thought is required. Should we sequence just the exomes? Should we perform targeted follow-up rather than genome-wide sequencing? The issue now becomes one of study design. For many groups, data from GWAS have already been collected, and several SNPs have been implicated as being associated with phenotype. The desire is to refine that signal, by hunting in regions around those "index" SNPs. However, exactly how should such a study be designed? Assuming we can't sequence everybody everywhere, which sample members should be sequenced, and where? How should the targeted sequencing regions be defined? Should we just sequence the LD block containing the index SNP? Should we include an additional few hundred kb to either side of the index SNP? Should some more principled procedure be used to define regions?

These questions of study design are key, but good guidance is hard to find. Two possible strategies present themselves for deciding between study designs: simulation-based, or databased.

\section{Simulation-Based Exploration of Study Design}

Here, we imagine simulating data under a range of possible scenarios that will provide intuition regarding the study design issues outlined above. Such a strategy involves many steps:

i) Generate a population of genotypes, $G$. (Here one might use a coalescent model or re-sampled observed data, such as from the 1000 Genomes Project.)

ii) Generate disease phenotypes conditional on one or more SNPs within the region of interest. (Here, one has to decide what disease model to simulate.)

iii) Model the first-stage association study (including the choice of subset $G_{1}$ to act as the sample, choice of tagSNPs, and calculation of test stats; here, we also have to condition on finding associated SNPs when test statistics are calculated.)

iv) Define the regions and depth of sequencing.

v) Select individuals, $G_{2}$, to include in the second-stage sequence analysis.

vi) Simulate the generation of sequence data for $\mathrm{G}_{2}$, including generation of sequence "reads", subsequent genotyping, alignment, and incorporated errors.

vii) Impute data, both within $\mathrm{G}_{2}$ where sites in the target region were not sequenced with adequate coverage for genotyping and for individuals in $\mathrm{G}_{1}$ not chosen to be in $\mathrm{G}_{2}$.

viii) Conduct an analysis of the resulting data.

The advantage of using a simulation-based scheme is that we can explore a wide range of scenarios for disease model and study design. Thus, replication is not a problem, and this will aid the ability to draw broader conclusions from the study. However, there are also significant drawbacks. As seen above, simulation studies involve many, non-trivial steps, and are, therefore, not straightforward to undertake. Secondly, we are forced to take a number of views regarding the models to use for the genotype-phenotype map, generation of sequence data, genotyping and mapping, etc. As George Box famously said, 
"All models are wrong; some are useful." The usefulness of these models is likely to be a function of how close they are to reality, and the state of reality is unknown. Nonetheless, despite these caveats, simulation-based studies clearly offer potential insights, and a number of papers have begun to appear.

This is the approach taken by Thomas et al. [34] to explore two-phase and family-based designs for sequencing studies. Taking the WECARE study of second breast cancers in relation to radiotherapy and DNA damage response genes [35] as an example of the former, and the Colorectal Cancer Family Study [36] as an example of the latter, we generated a virtual population of 10,000 $250 \mathrm{~kb}$ haplotypes by coalescent simulation (step i above). We then sampled individuals from this population and assigned them phenotypes (dropping genes through pedigrees for the family-based design) to generate replicate study samples (steps ii and iii in the above). First stage samples that met the ascertainment criteria (e.g., an association with a common variant for the two-phase design, multiple affected members for the family-based design) were then retained and a subsample of individuals were selected in various ways for the second stage (steps iv and $\mathrm{v}$ of the above). For example, for the two-phase design, individuals were selected by stratified sampling conditional on both disease status and carrier status of the associated SNP; for the family-based design, two or more affected relatives with various degrees of relationship to the proband, and possibly one or more unaffected relatives, were selected. Analyses then combined the data from the main and substudies (step viii) and results were compared across replicates. These analyses demonstrated the improvement in power that is possible by informative sampling jointly on disease and genotype, and (for family-based designs) by incorporating phenotype information from the entire pedigree, and not just those with sequence data (essentially using familial relationships to obtain additional "free" sequence data). In another example of the simulation-based approach applied to sequencing studies using DNA pooling, Liang et al. [37] explored the optimal trade-offs between sample size, numbers of pools, and depth of sequencing under realistic cost constraints.

\section{Data-Based Exploration of Study Design}

A second strategy is to examine existing data, in which samples have been sequenced as a follow-up to GWAS, and learn the appropriate lessons regarding the efficiency of study design. The National Institutes of Health decided to follow this approach in 2009 and released HG-09-014, "Comparing Design Approaches for Sequencing Disease-Associated Regions found in GWAS". This resulted in a consortium, GWASeq, in which a number of groups were funded to generate targeted sequence-level data in a follow-up to existing GWAS hits. Those groups were based at the Broad
Institute, Harvard University, University of Iowa, University of Michigan, and the University of Southern California (USC). The phenotypes studied included two cancers (breast and colon), cleft-lip/palate, psoriasis, and inflammatory bowel disease. All studies included population-based GWAS data, but two (those from University of Iowa and USC) also contained family-based data. Each group provided sample material for sequencing, and those samples were sequenced in a number of regions. In our own (USC) study, for example, we sequenced ten regions, resulting in a total of $5 \mathrm{MB}$ of sequenced genome per sample. Typical sequencing depths were of the order of 60-80x, and around 4,000 samples were sequenced [manuscript in preparation]. Loosely speaking, the strategy (in the case of the USC study, at least) is to generate data that are richer than might ordinarily be the case, in the sense of sequencing wider regions, at greater depth, and for more samples, and to then explore possible subsets of that design and determine how much of the signal resulting from the full data is lost. For example, we are currently subsampling the raw read data from the USC GWASeq project to assess the optimal trade-off between sample size and depth of sequencing.

All GWASeq consortium members are committed to uploading the resulting sequence data to $\mathrm{dbGaP}$. As such, their projects promise to provide an excellent resource for groups wishing to use existing experimental data to explore the efficacy of differing possible study designs.

\section{Use of Biological Information}

One way of using external biological information to improve analyses of GWAS and/or sequence-level data is to use it to describe the genetic networks that underpin the phenotypes. Another is to use it to inform underlying prior information within the analysis. We describe these below.

\section{Modeling of Genetic Networks}

Genetic polymorphisms, such as SNPs, do not typically affect phenotype directly. Rather, a SNP might affect the rate at which a gene is expressed, which will have a downstream effect on phenotype. In other words, a SNP might more reasonably be expected to affect parameters of the underlying Gene Regulatory Networks [GRNs] that result in a given phenotype. Alternatively, the SNP might affect the actual structure of that network.

By analyzing the effect of genetic polymorphism on GRN parameters, rather than directly on the resulting clinical phenotype, we bring the study phenotype closer to the polymorphism itself. As such, the signal is likely to be stronger, and power will be gained. For a discussion of this approach see 
[38], for example. As an example of the potential gains of such an approach, Wang et al., simulated data relating to two GRNs in a mouse heart cell model [39]. Crucially, besides measuring the final phenotypes, they also assumed that one could directly measure the parameters of the underling GRNs. They then conducted two GWAS-style analyses: one in which they tested for SNPs associated with resulting cellular phenotype; and another in which they tested for SNPs associated with changes to underlying parameter values. (In the simulation model, SNPs were assumed to affect network parameters directly.) They found that when testing for association with network parameters, the mean detection rate for associated SNPs was approximately two orders of magnitude higher than when testing for association with cellular phenotypes. Furthermore, the amount of phenotypic variation explained was 30-40\% when using the set of SNPs identified as associated with underlying network parameters, compared to 5-20\% when using SNPs identified as associated with cellular phenotype.

The caveat with the assumptions of Wang et al. [39], of course, is that one is not typically able to measure directly GRN parameters. This suggests that some future effort might be well spent in estimating the underlying GRNs, inferring (or, ideally, observing) the changes to parameters between cases and controls, and then conducting association tests on the network parameters directly.

A growing literature is appearing in which attempts are made to reconstruct underlying genetic networks. These approaches take either a "top-down" or "bottom-up" approach. Top-down approaches attempt to infer the GRN from data such as gene expression levels. A popular such approach involves the use of Dynamic Bayesian Networks [DBNs] [e.g., 40•, 41]; it can flexibly and easily deal with real-life complications such as noise and missing data, is hierarchical in nature, and provides a framework for formal inference [e.g., $42,43]$. In contrast, bottom-up approaches attempt to build models using detailed descriptions of underlying biochemical processes. An example of this approach can be found in Kholodenko et al. [44].

One problem that arises in this context is that networks might be of sufficient complexity to make explicit calculation of downstream phenotype intractable. This is an illustration of a phenomenon that is becoming common, that is, as the size and complexity of modern datasets grow, analytic tractability is often lost. A common response to this is the use of "approximate Bayesian computation" $[\mathrm{ABC}]$. In these methods, calculation of likelihoods is replaced by a simulation step in which, loosely speaking, one constructs empirical estimates of the likelihood. For a nice survey of ABC methods see [45].

An example of the ABC approach to GRN analysis appeared in a recent paper by Rau et al. [46]. They used timecourse gene expression data to infer the structure and parameters of a GRN relating to a well-characterized pathway involving the Raf signaling protein in the human immune system. They also applied their method to the S.O.S. DNA repair system in $E$. coli. They showed that their method could infer GRN structure with some success, although currently only for small networks. Related work from the research group of Stumpf, et al. [e.g., 47] has also explored the application of $\mathrm{ABC}$ analysis to GRNs, and has resulted in the release of the software package $\mathrm{ABC}$-SysBio, which allows ABC analysis of GRNs described using the SysBio Markup language of Liepe et al. [48].

\section{Use of Biological Information to Inform Priors}

The idea of using hierarchical models to stabilize the estimates of many genetic risk parameters that are individually based on sparse data was mentioned earlier. The real power of hierarchical modeling, however, derives from its ability to incorporate external information, such as genomic or pathway annotations, into the analysis of the data at hand in a principled manner. For example, one might take the log relative risk $\beta_{v}$ for each variant $v$ as the dependent variable in a second-level regression on a vector of explanatory variables, such as whether they are coding or regulatory variants, their evolutionary conservation or predicted effects on protein conformation [49]. Used in this manner, the external information does not provide a specific prior distribution for the RR parameters but allows the ensemble of all the data to determine whether this information is useful and allows for the uncertainty about its magnitude to be properly accounted for in assessing the posterior distribution of the RRs. While the obvious way to do this is through a simple linear regression of the expectation of lnRR on these prior covariates, this assumes these secondlevel associations are in a consistent direction. Other possibilities might put these prior covariates in a model for the variance (in the spirit of the $\mathrm{C}_{\alpha}$ test) or use prior gene-gene connection information (such as from gene co-expression networks) in a model for the covariances of the lnRRs [50]. Yet another possibility, in the spirit of burden tests, is to assign each variant a weight of $+1,-1$, or 0 in an index that simply sums the allele counts, with probabilities that depend upon these prior covariates, thereby allowing for uncertainty about the direction of effect for any given variant or if the variant has any effect at all $[51,52 \bullet, 53]$.

However they are to be used, there are many sources of such prior information. Various databases such as the Gene Ontology provide expert-curated information about single variants, genes, or pathways [54]. Various programs are available to provide predictions of functional significance, such as SIFT [55], PolyPhen [56], and FunciSNP [57]. Actual measurement of biological activity of specific variants may be obtained from in vitro assays, such as expression data from the GTEx project [58]. 


\section{Conclusions}

Sequence-based follow-up to GWAS is clearly a strategy that has many appealing features. However, it is also clear that we are very much in the early stages of learning how to deal with such sequence data. Methods of analysis for rare variants are rapidly evolving, and we can expect to see many publications emerging soon that will provide evidence about the relative contribution of rare variants to the missing heritability. Nevertheless, the enthusiasm for this new technology, coupled with the considerable investments that have already been made, implies that more thought is warranted regarding the most efficient use of limited resources (samples and money). While there have been some developments in study design for next-generation sequencing studies, reviewed above, there remain many open questions: what are the relative merits of family-based vs. population-based designs? What is the appropriate trade-off between sample size and depth of sequencing? Who should be selected for sequencing based on already available phenotype and genotype information (and for family studies, familial relationships)? How can we best exploit external information? What should we do about replication? These kinds of questions are rich fodder for methodological research, using either the simulation-based approach or subsampling from real data as we've described.

Acknowledgements The authors gratefully acknowledge funding from NIH grants GM103804, HG005927, HG002790, ES020794, and CA148107. The views in this article are those of the authors, and do not necessarily reflect the views of NIH.

\section{Compliance with Ethics Guidelines}

Conflict of Interest P Marjoram and DC Thomas both declare no conflicts of interest.

Human and Animal Rights and Informed Consent This article does not contain any studies with human or animal subjects performed by any of the authors.

\section{References}

Papers of particular interest, published recently, have been highlighted as:

- Of importance

-. Of major importance

1. Gudmundsson J, Sulem P, Manolescu A, Amundadottir LT, Gudbjartsson D, et al. Genome-wide association study identifies a second prostate cancer susceptibility variant at $8 \mathrm{q} 24$. Nat Genet. 2007;39:631-7.

2. McPherson R, Pertsemlidis A, Kavaslar N, Stewart A, Roberts R, et al. A common allele on chromosome 9 associated with coronary heart disease. Science. 2007;316:1488-91.
3. Rioux JD, Xavier RJ, Taylor KD, Silverberg MS, Goyette P, et al. Genome-wide association study identifies new susceptibility loci for Crohn disease and implicates autophagy in disease pathogenesis. Nat Genet. 2007;39:596-604.

4. Saxena R, Voight BF, Lyssenko V, Burtt N, de Bakker PI, et al. Genome-wide association analysis identifies loci for type 2 diabetes and triglyceride levels. Science. 2007;316:1331-6.

5. Sladek R, Rocheleau G, Rung J, Dina C, Shen L, et al. A genomewide association study identifies novel risk loci for type 2 diabetes. Nature. 2007:445:881-5.

6. Scott LJ, Mohlke KL, Bonnycastle LL, Willer CJ, Li Y, et al. A genome-wide association study of type 2 diabetes in Finns detects multiple susceptibility variants. Science. 2007;316:1341-5.

7. Steinthorsdottir V, Thorleifsson G, Reynisdottir I, Benediktsson R, Jonsdottir T, et al. A variant in CDKAL1 influences insulin response and risk of type 2 diabetes. Nat Genet. 2007;39:770-5.

8. Zeggini E, Weedon MN, Lindgren CM, Frayling TM, Elliott KS, et al. Replication of genome-wide association signals in UK samples reveals risk loci for type 2 diabetes. Science. 2007;316:1336-41.

9. Lango Allen H, Estrada K, Lettre G, Berndt SI, Weedon MN, et al. Hundreds of variants clustered in genomic loci and biological pathways affect human height. Nature. 2010;467:832-8.

10. Macgregor S, Cornes BK, Martin NG, Visscher PM. Bias, precision and heritability of self-reported and clinically measured height in Australian twins. Hum Genet. 2006;120:571-80.

11. Silventoinen K, Sammalisto S, Perola M, Boomsma DI, Cornes BK, et al. Heritability of adult body height: a comparative study of twin cohorts in eight countries. Twin Res. 2003;6:399-408.

12. Visscher PM, Hill WG, Wray NR. Heritability in the genomics eraconcepts and misconceptions. Nat Rev Genet. 2008;9:255-66.

13. Figueiredo J, Stram D, Haiman C. The Impact of GWAS Findings on Cancer Etiology and Prevention. Current Epidemiology Reports 2014: 1-8. This reviews the implications of GWAS for screening strategies and genetically-targeted pharmacogenetics and discusses new research directions in the post-GWAS era.

14. Dickson SP, Wang K, Krantz I, Hakonarson H, Goldstein DB. Rare variants create synthetic genome-wide associations. PLoS Biol. 2010;8:e1000294.

15. Sun X, Namkung J, Zhu X, Elston RC. Capability of common SNPs to tag rare variants. BMC Proc. 2011;5:S88.

16. Purcell SM, Wray NR, Stone JL, Visscher PM, O'Donovan MC, et al. Common polygenic variation contributes to risk of schizophrenia and bipolar disorder. Nature. 2009;460:748-52.

17. Yang J, Benyamin B, McEvoy BP, Gordon S, Henders AK, et al. Common SNPs explain a large proportion of the heritability for human height. Nat. Genet. 2010:565-9.

18. Hunt KA, Mistry V, Bockett NA, Ahmad T, Ban M, et al. Negligible impact of rare autoimmune-locus coding-region variants on missing heritability. Nature. 2013;498:232-5. This paper calls into question the hypothesis that the missing heritability may be attributable to rare coding variants by targeted exome resequencing of a very large sample of cases and controls and suggests that many common variants with small effect sizes may have a larger causal contribution.

19.•• Visscher PM, Brown MA, McCarthy MI, Yang J. Five Years of GWAS Discovery. Am J Hum Genet. 2012;90:7-24. This is an excellent review of the accomplishments of GWAS, including novel biological insights, clinical relevance, and interpretation of associations.

20. Madsen BE, Browning SR. A groupwise association test for rare mutations using a weighted sum statistic. PLoS Genet. 2009;5: e1000384.

21. Neyman J, Scott E. On the use of c(alpha) optimal tests of composite hypotheses. Bull Internat Statist Inst. 1966;41:477-97.

22. Neale BM, Rivas MA, Voight BF, Altshuler D, Devlin B, et al. Testing for an unusual distribution of rare variants. PLoS Genet. 2011;7:e1001322. 
23. Wu MC, Lee S, Cai T, Li Y, Boehnke M, et al. Rare-variant association testing for sequencing data with the sequence kernel association test. Am J Hum Genet. 2011;89:82-93.

24. Lee $\mathrm{S}, \mathrm{Wu} \mathrm{MC}$, Lin $\mathrm{X}$. Optimal tests for rare variant effects in sequencing association studies. Biostatistics. 2012;13:762-75.

25. Schaid DJ, McDonnell SK, Sinnwell JP, Thibodeau SN. Multiple Genetic Variant Association Testing by Collapsing and Kernel Methods With Pedigree or Population Structured Data. Gen Epidemiol. 2013;37:409-18. This paper provides a general kernel machine regression framework for testing multiple rare variants in both pedigrees and case-control samples of unrelated individuals, including structured populations.

26. Chen H, Meigs JB, Dupuis J. Sequence Kernel Association Test for Quantitative Traits in Family Samples. Genet Epidemiol. 2013;37: 196-204.

27. Ionita-Laza I, Lee S, Makarov V, Buxbaum JD, Lin X. Familybased association tests for sequence data, and comparisons with population-based association tests. Eur J Hum Genet. 2013;10: $1158-62$.

28. Witte JS. Genetic analysis with hierarchical models. Genet Epidemiol. 1997; 14:1137-42.

29.• Zuk O, Hechter E, Sunyaev SR, Lander ES. The mystery of missing heritability: Genetic interactions create phantom heritability. Proc Nat Acad Sci. 2012;109:1193-98. This paper provides an excellent exploration of the issue of missing heritability, from the perspective of the definition of heritability itself.

30. Lewinger JP, Morrison JL, Thomas DC, Murcray CE, Conti DV, et al. Efficient two-step testing of gene-gene interactions in genomewide association studies. Genet Epidemiol. 2013;37:440-51.

31. Baurley JW, Conti DV. A scalable, knowledge-based analysis framework for genetic association studies. BMC Bioinformatics. 2013;14:312.

32. Baurley JW, Conti DV, Gauderman WJ, Thomas DC. Discovery of complex pathways from observational data. Stat Med. 2010;29: 1998-2011.

33. Chen GK, Thomas DC. Using biological knowledge to discover higher order interactions in genetic association studies. Genet Epidemiol. 2010;34:863-78.

34. Thomas DC, Yang Z, Yang F. Two-Phase and Family-Based Designs for Next-Generation Sequencing Studies. Front Gen. 2013;4:Art276.

35. Bernstein JL, Langholz B, Haile RW, Bernstein L, Thomas DC, et al. Study design: evaluating gene-environment interactions in the etiology of breast cancer - the WECARE study. Breast Cancer Res. 2004;6:199-214.

36. Newcomb P, Haile R, Anton-Culver H, Gallinger S, Hopper J, et al. The Colorectal Cancer Family Registry: 1998-2002. Cancer Epidemiol Biomarkers Prev. 2002;11:1222s.

37. Liang WE, Thomas DC, Conti DV. Analysis and Optimal Design for Association Studies Using Next-Generation Sequencing With Case-control Pools. Genet Epidemiol. 2012;36:870-81.

38. Marjoram P, Zubair A, Nuzhdin SV. Post-GWAS: where next? More samples, more SNPs or more biology? Heredity. 2014;112: 79-88.

39. Wang Y, Gjuvsland AB, Vik JO, Smith NP, Hunter PJ, et al. Parameters in Dynamic Models of Complex Traits are Containers of Missing Heritability. PLoS Comput Biol. 2012;8:e1002459.

40. Penfold CA, Wild D. How to infer gene networks from expression profiles, revisited. J R Soc Interface Focus. 2011;6:857-70. This is one in a series of papers that develop methods for analysis of gene regulatory networks from a Bayesian perspective.

41. Penfold C, Buchanan-Wollaston V, Denby K, Wild D. Nonparametric Bayesian inference for perturbed and orthologous gene regulatory networks. Bioinformatics. 2012;28:233-41.
42. Breeze E, Harrison E, McHattie S, Hughes L, Hickman R, et al. High resolution temporal profiling of transcripts during Arabidopsis leaf senescence reveals a distinct chronology of processes and regulation. Plant Cell. 2011;23:873-94.

43. Windram O, Madhou P, McHattie S, Hill C, Hickman R, et al. Arabidopsis defense against botrytis cinerea: chronology and regulation deciphered by high-resolution temporal transcriptomic analysis. Plant Cell Online. 2012;24:3530-57.

44. Kholodenko BN, Kiyatkin A, Bruggeman FJ, Sontag E, Westerhoff $\mathrm{HV}$, et al. Untangling the wires: a strategy to trace functional interactions in signaling and gene networks. Proc Natl Acad Sci. 2002;99:12841-6.

45. Beaumont MA. Approximate Bayesian Computation in Evolution and Ecology. Annu Rev Ecol Evol Syst. 2010;41:379-406.

46. Rau A, Jaffrézic F, Foulley J-L, Doerge RW. Reverse engineering gene regulatory networks using approximate Bayesian computation. Stat Comput. 2011;22:1257-71.

47. Secrier M, Toni T, Stumpf MPH. The ABC of reverse engineering biological signalling systems. Mole Biosystems. 2009;5:1925.

48. Liepe J, Barnes C, Cule E, Erguler K, Kirk P, et al. ABC-SysBioapproximate Bayesian computation in Python with GPU support. Bioinformatics. 2010;26:1797-9.

49. Lewinger JP, Conti DV, Baurley JW, Triche TJ, Thomas DC. Hierarchical Bayes prioritization of marker associations from a genome-wide association scan for further investigation. Genet Epidemiol. 2007;31:871-82.

50. Thomas DC, Conti DV, Baurley J, Nijhout F, Reed M, et al. Use of pathway information in molecular epidemiology. Hum Genomics. 2009; 4:21-42.

51. Quintana MA, Berstein JL, Thomas DC, Conti DV. Incorporating model uncertainty in detecting rare variants: the Bayesian risk index. Genet Epidemiol. 2011;35:638-49.

52. Quintana MA, Schumacher FR, Casey G, Bernstein JL, Li L, et al. Incorporating prior biologic information for high-dimensional rare variant association studies. Hum Hered. 2012;74:184-95. This extends the Bayesian hierarchical modeling framework to rare variants by incorporating a model for variant selection into a burden index leveraging external genomic annotation information, with applications to variants of unknown significance in BRCA1 for breast cancer and to multiple genes in DNA repair pathways for colon cancer.

53. Quintana MA, Conti DV. Integrative variable selection via Bayesian model uncertainty. Stat Med. 2013;32:4928-53.

54. Conti DV, Lewinger JP, Swan GE, Tyndale RF, Benowitz $\mathrm{NL}$, et al. Using ontologies in hierarchical modeling of genes and exposures in biologic pathways. In Swan GE, editors. Phenotypes and Endophenotypes: Foundations for Genetic Studies of Nicotine Use and Dependence, 2009, NCI Tobacco Control Monographs No. 20: Bethesda, MD. pp. 539-84.

55. Ng PC, Henikoff S. SIFT: Predicting amino acid changes that affect protein function. Nucleic Acids Res. 2003;31:3812-4.

56. Xi T, Jones IM, Mohrenweiser HW. Many amino acid substitution variants identified in DNA repair genes during human population screenings are predicted to impact protein function. Genomics. 2004;83:970-9.

57. Coetzee SG, Rhie SK, Berman BP, Coetzee GA, Noushmehr H. FunciSNP: an R/bioconductor tool integrating functional non-coding data sets with genetic association studies to identify candidate regulatory SNPs. Nucleic Acids Res. 2012;gks542.

58. Lonsdale J, Thomas J, Salvatore M, Phillips R, Lo E, et al. The Genotype-Tissue Expression (GTEx) project. Nat Genet. 2013;45: $580-5$. 